\title{
Genetic Etiology of Tumors with Genome-Wide Association Study
}

\author{
Ming Zheng ${ }^{1, a^{*}}$ and Mugui Zhuo ${ }^{1, b^{*}}$ \\ ${ }^{1}$ Guangxi Colleges and Universities Key Laboratory of Professional Software Technology, Wuzhou \\ University, Wuzhou, China \\ a370505375@qq.com, b756456050@qq.com \\ *The Corresponding author
}

Keywords: Genome wide association study; Tumors; genetic factors; Etiology

\begin{abstract}
Cancer is a complex disease caused by gene-environment interaction. In the same environmental exposure, the risk of individuals with different genetic backgrounds of cancer is very different. The related factors of tumor genetic research have important significance in understanding the genesis and development of tumor diagnosis and treatment. A genome-wide association study in recent years, the development of genome-wide (association study, GWAS) can be found that genetic factors associated with complex disease or phenotype in whole genome range, provides a powerful means for the study of complex disease genetics. Researchers in the United States and Europe using genome-wide closed method research, on a variety of common cancers studied, has obtained important results in. 2010, China scientists published a series of high the level of tumor genome-wide association study results in international core journals, the Chinese common tumor in genetic disease Significant progress has been made in the field of scientific research.
\end{abstract}

\section{Introduction}

Scientific research shows that nearly a hundred years, cancer is a complex disease caused by gene environment interaction, but in the same environmental exposure, the risk of individual cancer are very different[1]. Some people never had cancer, while others in their life is suffering from cancer, genetic difference and the individual susceptibility factors. Therefore, the genetic etiology of cancer to reveal the mechanism of cancer, cancer of the individual prevention is of great significance[2]. In addition, an important factor in cancer genetic susceptibility factors are cancer progression, so to reveal the genetic susceptibility factor also has an important significance for cancer through clinical treatment and prognosis. Observation and long-term basic research, the vast majority of inherited cancer susceptibility genes have been revealed. These hereditary cancer is "cancer syndrome" by Dan Jiyin Germline mutations cause, such as the BRCA1 gene germline mutation caused by breast cancer and ovarian cancer. However, a single gene germline mutation caused by "cancer syndrome" is extremely rare in the general population, the majority of these cancers are not susceptible gene germline mutations, but by genetic variation and environmental factors. The identification of these genetic variants associated with cancer is nearly 20 years of hot research topic, research methods from the previous single gene association studies to genome-wide association studies (genome-wide association study, GWAS)[3,4].

GWAS is the use of high density biochip, on a genome-wide of single nucleotide polymorphism (SNP) screening, the frequency difference between cases and controls, and then verified by large amount of samples, so as to obtain the research methods and the most significant disease or trait related genes or mutations in the genome wide[5]. The study method is abandoned the previous candidate gene method to the study of the pre human hypothesis, this hypothesis requires very understanding of the relationship between gene and disease, and in fact the relationship often is not very clear. GWAS without prior assumptions, but in the whole genome range of comprehensive and systematic screening. At the same time is generally GWAS using a large sample size (thousands or even tens of thousands of cases and controls) and test standard strictly, independent sample to verify the positive results obtained even after the multi center, so the research results are reliable. The 
western developed countries scientists on cancer, diabetes, cardiovascular disease, rheumatism, mental illness and other complex diseases, as well as height body mass index, blood lipid levels and other complex traits has carried out many studies. According to statistics, as of the end of 2013, a total of Nearly 2000 papers published in GWAS, reported nearly 6000 traits and more than 14000 traits related to SNP, has made remarkable achievements[6].

\section{Three Kinds of Cancers}

Genetic Etiology of Esophageal Squamous Cell Carcinoma. Esophageal squamous cell carcinoma (the esophagus) is a common malignant tumor of digestive tract, in cancer death fourth in China. Around the world each year about 300 thousand new cases, more than half of them occurred in Chinese. Esophageal cancer insidious onset, most patients have been in advanced stage, poor treatment effect, 5-year survival rate about $20 \%$. therefore, carry out esophageal cancer susceptibility gene GWAS, found that the prevention of esophageal cancer is of particular importance for.

The study also found that the gene environment interaction was 3 SNP and smoking in $12 q 24$ and drinking, the chromosomal regions containing aldehyde dehydrogenase 2 gene involved in the metabolism of alcohol (ALDH2). Further research and analysis of the GWAS data, and found 6 new susceptibility loci in esophageal carcinoma, which is located in 4q23, 16q12.1, 22q12 and 3q27 4 SNP and the risk of esophageal cancer showed a marginal effect significantly 2 and SNP at 2 q22 and $13 \mathrm{q} 33$ in gene drinking interaction mode is associated with an increased risk of esophageal cancer. In addition, alcohol dehydrogenase (ADHs) gene cluster 4q23 susceptibility loci with significant interaction was further confirmed by the metabolism of alcohol drinking, genetic variation and susceptibility to esophageal cancer previously reported. Genetic variation combined with ADH1B and ALDH2 and analyze whether drinking found, carrying the risk genotype if drinking, the risk of esophageal cancer is about 4 times higher than non-drinkers, that gene environment interaction is very important to the national[7].

There are two major susceptibility genes or mutations in cancer research to elucidate the molecular basis for susceptibility to a phenomenon, explain the heterogeneity among cancer, two is for the identification of susceptible populations for early warning and prevention of individual selection of effective biomarkers. We divide the experiment 17 Department of esophageal cancer GWAS the susceptible loci and 8 showed gene environment interaction susceptibility loci as well as gender, age, smoking and drinking 4 non genetic factors, establish the prediction model of the risk of esophageal cancer. The results show that the prediction model by the 25 SNP and gender, age, smoking and drinking of esophageal cancer, can prediction of $70 \%$ esophageal cancer patients, compared with non-genetic factors to establish the model of improved $7 \%$, indicating that the increase of genetic susceptibility factors contributes to improving the effectiveness of esophageal cancer risk prediction. In addition to the study and esophageal cancer Genetic factors associated with the prognosis of GWAS, genetic variation and Strategy Research of tumor patients and is currently a hot topic. We conducted a genome-wide association analysis for more than 5 years of follow-up of 1331 cases of patients with esophageal cancer, and had found the rs7242481G $>$ A variant of SLC39A6 gene 5 '-UTR' length of survival, with the median survival time of AA the genotype of the patients was 16 months, and with the GG genotype of the patients was 32 months. The results have been verified in from Jiangsu and Guangzhou a total of 1962 cases of patients with esophageal cancer. To elucidate its mechanism, a series of biochemical and molecular biology function research, found that the variation of $\mathrm{G}>\mathrm{A}$ damage a suppression of SLC39A6 transcription factor binding sites, increase the expression of the gene, promote the proliferation and metastasis of esophageal carcinoma cells, so A Allele The study shows that SLC39A6 is an important molecular and prognostic marker for the progression of esophageal cancer[8]. The cancer genes results can be seen as Fig. 1. 


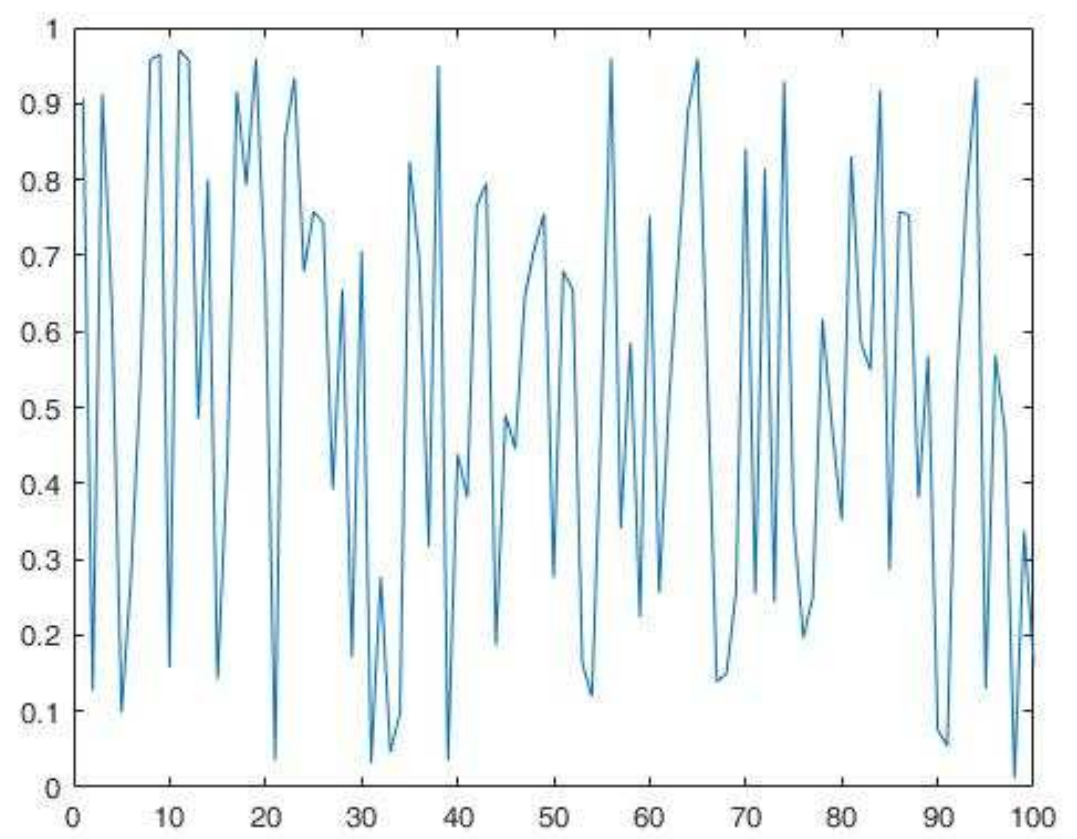

Figure 1. Significantly increased risk of Esophageal Squamous Cell Carcinoma happens with the number of risk genes

Genetic Etiology of Lung Cancer. Lung cancer is one of the most common malignant tumors, the first tumor mortality. China ranked the United States and Europe have carried out a number of lung cancer GWAS, found some important predisposing loci, some susceptible genes exist in Chinese population. Due to the different environment and population genetic background, Chinese people may have different with western populations of lung cancer susceptible sites, so it is necessary for us to study. In the East Asian countries including Chinese, common nonsmoking female lung adenocarcinoma. In order to explain the genetic mechanism and compared with smoking related lung cancer, the National Cancer Institute and Chinese multiple units, the Asian non-smoking female lung cancer GWAS. the screening study the China, Hong Kong, Taiwan, Japan, South Korea and Singapore, a total of 5510 cases of nonsmoking female lung cancer patients and 4544 cases of control The whole genome association and the genetic variation of lung cancer, and in 1099 cases of nonsmoking female lung cancer patients and 2913 cases to verify the control, 10q25.2, 6q22.2 and 6 p21.32 found other susceptibility loci of 3 nonsmoking female lung cancer. It is demonstrated that the susceptibility loci previously reported by GWAS 5p15.33, 3q28 and 17q24.3 3 a proof of lung cancer, susceptibility loci in $15 \mathrm{q} 25$ significantly increased the risk of lung cancer in smokers. In addition to genetic variation associated with pathogenesis of lung cancer, there are several methods for the use of GWAS and lung cancer genetic variation in sensitivity, prognosis of chemotherapy. There is relationship between genome SNP and small cells lung cancer patients receiving platinum based chemotherapy sensitivity and survival, found that the YAP1 gene was significantly associated with the survival time of SNP, the function of experiments suggest that the SNP can regulate YAP1 expression, thus affecting the prognosis of patients with small cell lung cancer ${ }^{[9]}$.

Genetic Etiology of Viral Infection Related Cancers. Hepatocellular carcinoma (HCC)[10] is one of the most common tumor Chinese, mortality ranked third of cancer deaths, hepatocellular carcinoma occurred in about $80 \%$ of the world's China, hepatitis B virus (HBV) infection is the main cause. However, HBV is also a chronic infection, develop liver cancer patients to $1 / 5$, suggesting that genetic susceptibility plays an important role in the occurrence and development of hepatocellular carcinoma. To reveal the genetic susceptibility factors will contribute to the treatment of hepatitis HBV and liver cancer individualized prevention. To reveal the individual genetic predisposing factors 
for elucidating the carcinogenic mechanism and prevention of these viruses is obviously very important.

\section{Summary}

GWAS mission is to find the susceptible genes and the virulence genes, but the function and mechanism of reliability not only for further verification of the results, and the subsequent application in cancer are crucial. Finally, and most important, is to study how to apply the research results to practice, which should be used for individual cancer early warning, prevention, intervention and treatment. All the work is challenging, but also a great opportunity, hope our scientists can make a breakthrough in this research field, and make greater contribution to the China tumor genetic etiology.

\section{Acknowledgements}

This work was supported by grants from The National Natural Science Foundation of Chi-na (No. 61502343, No. 61373051, and No. 61402423), China Postdoctoral Science Foun-dation funded(No. 2016M590260), the Guangxi Natural Science Foundation (No. 2015GXNSFBA139262), the Science Research Funds for the Guangxi Universities (No. KY2015ZD122), Guangxi Colleges and Universities Key Laboratory of Professional Software Technology, Wuzhou University.

\section{References}

[1] YU L L, LU M Y, JIA D Y, et al. Modeling the Genetic Regulation of Cancer Metabolism: Interplay between Glycolysis and Oxidative Phosphorylation[J]. Cancer Research, 2017, 77(7): 1564-1574.

[2] LENGAUER C, KINZLER K W, VOGELSTEIN B. Genetic instabilities in human cancers[J]. Nature, 1998, 396(6712): 643-649.

[3] AFSHARI N A, IGO R P, MORRIS N J, et al. Genome-wide association study identifies three novel loci in Fuchs endothelial corneal dystrophy[J]. Nature Communications, 2017, 8.

[4] HOLLMANN A K, BLEYER M, TIPOLD A, et al. A genome-wide association study reveals a locus for bilateral iridal hypopigmentation in Holstein Friesian cattle[J]. Bmc Genetics, 2017, 18.

[5] BURTON P R, CLAYTON D G, CARDON L R, et al. Genome-wide association study of 14,000 cases of seven common diseases and 3,000 shared controls[J]. Nature, 2007, 447(7145): 661-678.

[6] MCCARTHY M I, ABECASIS G R, CARDON L R, et al. Genome-wide association studies for complex traits: consensus, uncertainty and challenges[J]. Nature Reviews Genetics, 2008, 9(5): 356-369.

[7] QIAO Y, LI J, SHI C, et al. Prognostic value of circulating tumor cells in the peripheral blood of patients with esophageal squamous cell carcinoma[J]. Onco Targets Ther, 2017, 10: 1363-1373.

[8] JI H, CAO M, REN K, et al. Expression and Clinicopathological Significance of Mel-18 and Bmi-1 in Esophageal Squamous Cell Carcinoma[J]. Technol Cancer Res Treat, 2017: 1533034617705055.

[9] ABDEL-RAHMAN O. Causes of death in long-term lung cancer survivors: a SEER database analysis[J]. Curr Med Res Opin, 2017: 1-16.

[10] RICH N E, PARIKH N D, SINGAL A G. Hepatocellular Carcinoma and Liver Transplantation: Changing Patterns and Practices[J]. Curr Treat Options Gastroenterol, 2017. 